\title{
Modular Relative Jacobian for Dual-Arms and the Wrench Transformation Matrix
}

\author{
Rodrigo S. Jamisola Jr., Petar Kormushev, Darwin G. Caldwell and Frank Ibikunle
}

\begin{abstract}
A modular relative Jacobian is recently derived and is expressed in terms of the individual Jacobians of stand-alone manipulators. It includes a wrench transformation matrix, which was not shown in earlier expressions. This paper is an experimental extension of that recent work, which showed that at higher angular end-effector velocities the contribution of the wrench transformation matrix cannot be ignored. In this work, we investigate the dual-arm force control performance, without necessarily driving the end-effectors at higher angular velocities. We compare experimental results for two cases: modular relative Jacobian with and without the wrench transformation matrix. The experimental setup is a dual-arm system consisting of two KUKA LWR robots. Two experimental tasks are used: relative end-effector motion and coordinated independent tasks, where a force controller is implemented in both tasks. Furthermore, we show in an experimental design that the use of a relative Jacobian affords less accurate task specifications for a highly complicated task requirement for both end-effectors of the dual-arm. Experimental results on the force control performance are compared and analyzed.

Index Terms-Dual-arms, relative Jacobian, modular, wrench transformation matrix, force control, single manipulator control
\end{abstract}

\section{INTRODUCTION}

A relative Jacobian is used to control the dual-arm as a single manipulator (with one end-effector). This approach drastically increases the dimension of the combined null space, as compared to controlling each individual manipulators separately. For example, when each manipulator of a dual-arm has seven degrees-of-freedom (7-DOF) and are controlled in full Cartesian space individually, the combined null space has only 2-DOF. However, through the use of a relative Jacobian, the relative motion between the two end-effectors is considered such that at full Cartesian space control between the two end-effectors, an 8-DOF combined null space results.

The use of relative Jacobian [1], [2] has recently gained renewed attention because of the increased studies in dualarms control [3]-[9], to name a few. Two major advantages

R. S. Jamisola Jr. and F. Ibikunle are with Electrical, Electronics and Telecommunications Engineering Department, Botswana International University of Science and Technology (BIUST), Private Bag 16, Palapye, Botswana 10071 \{jamisolar, ibikunlef\}ebiust.ac.bw.

P. Kormushev and D. Caldwell are with Department of Advanced Robotics, Istituto Italiano di Tecnologia (IIT), Via Morego 30, 16163 Genova, Italy \{petar.kormushev, darwin. caldwell\}ait.it.

This research was partially supported by the PANDORA EU FP7-Project under the Grant Agreement No. ICT-288273.

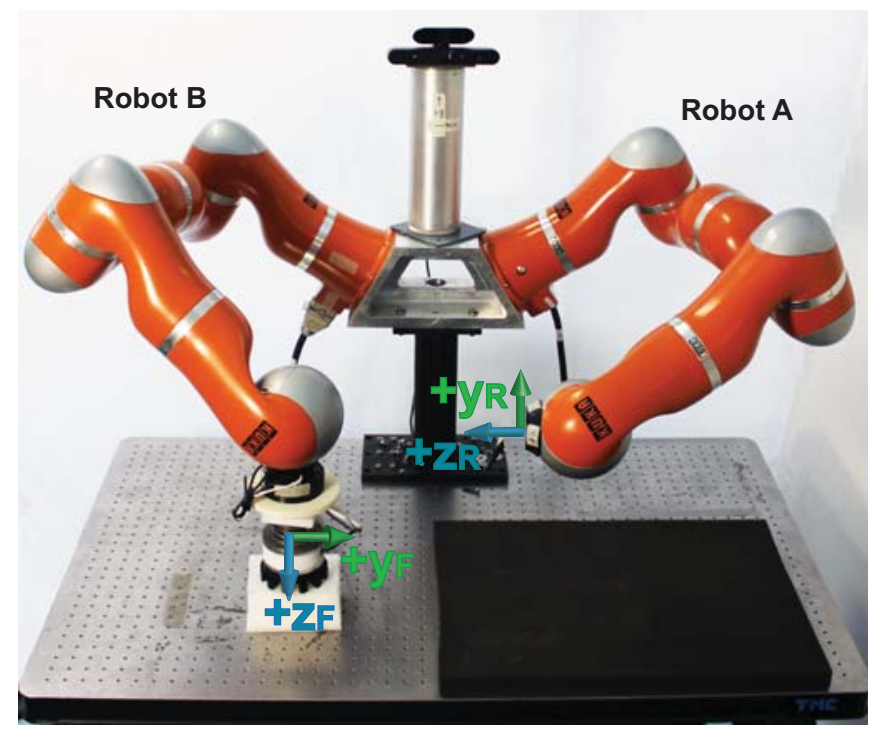

Fig. 1. The KUKA dual-arm manipulator setup. The right arm (robot $B$ ) end-effector holds the tool, while the left $\operatorname{arm}(\operatorname{robot} A)$ end-effector is the reference end-effector. Axes $\mathbf{z}_{R}$ and $\mathbf{y}_{R}$ are the relative position axes located at the robot $A$ end-effector frame, while $\mathbf{z}_{F}$ and $\mathbf{y}_{F}$ are the force sensor axes located at the force sensor frame. The tool end-effector exerts a normal force on the table (along $\mathbf{z}_{F}$ ), while the two end-effectors move relative to each other (horizontally along $\mathbf{z}_{R}$ ).

can be enumerated: (1) all the principles of single manipulator control can be applied to dual-arm manipulators, and (2) a dramatic increase in the null-space dimension. The first formulations of the relative Jacobian [1], [2] analytically computed it based on the assigned kinematic chain between the two end-effectors. In order to simplify its implementation, one approach is to express the relative Jacobian in modular form, that is, in terms of the Jacobian of each standalone manipulator.

Just recently, a newly derived modular relative Jacobian [10] reveals a wrench transformation matrix, which was not shown in previous expressions [11]-[13] or were in an unsimplified, non-compact form [14]-[16]. This new and recent expression drastically simplifies the relative Jacobian implementation because one only needs to consider the individual Jacobians for each manipulator, and then arrives at the relative Jacobian of the combined manipulators by performing corresponding Jacobian transformations as shown in [10]. In the same work, it was analytically proven that the exclusion of the wrench transformation matrix may result in an end-effector motion, for a null space motion when 


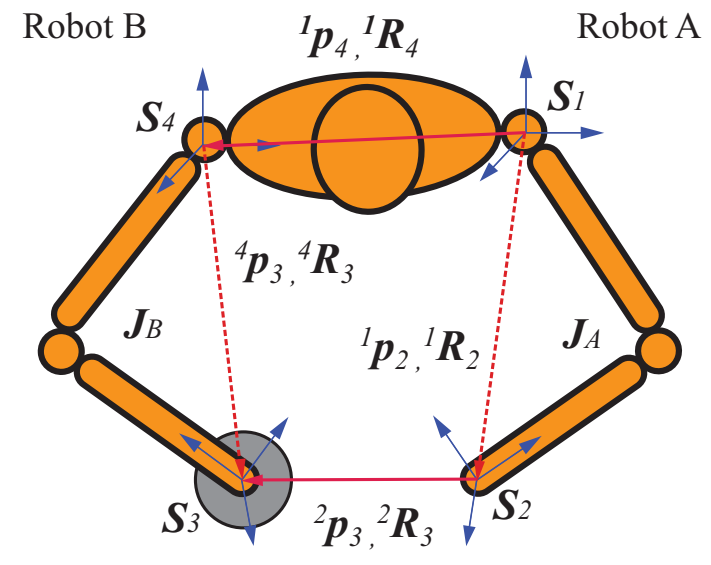

Fig. 2. Schematic diagram of the frame assignments for the dual-arm in Fig. 1. The tool end-effector (frame $\mathbf{S}_{3}$ ) is expressed with respect to the reference end-effector (frame $\mathbf{S}_{2}$ ). The corresponding position and orientation transformations are shown in the diagram. The Jacobian for robot $A$ is denoted $\mathbf{J}_{A}$ and the Jacobian for robot $B$ is denoted $\mathbf{J}_{B}$.

the wrench transformation is included. Furthermore, it was experimentally shown that at higher end-effector velocities, the effect of the wrench transformation matrix cannot be ignored.

This work extends the experimental investigation in [10] by evaluating a dual-arm force controller, without necessary driving the end-effectors at higher velocities. We investigate two cases: a modular relative Jacobian with (newly derived) and without (previously derived) the wrench transformation matrix. We perform two experimental tasks: relative endeffector motion and coordinated independent tasks. Both force and motion controllers [17]-[19] are implemented in full relative operational space for both experimental task executions. More recent studies on cooperative manipulation include trajectory planning for manipulation of flexible structure [20], manipulation and transportation of aerial robots [21], [22], decomposition frameworks with unilateral thrusters [23], and cooperative dual-arm mobile manipulation [24].

\section{THEORETICAL BACKGROUND}

To express the modular relative Jacobian $\mathbf{J}_{R}$ of a dual-arm, we let the Jacobian of the left arm (reference end-effector) to be $\mathbf{J}_{A}$, and the Jacobian of the right arm (tool end-effector) to be $\mathbf{J}_{B}$, as shown in Fig. 2. From the same figure, the position ${ }^{i} \mathbf{p}_{j}$ and rotation ${ }^{i} \mathbf{R}_{j}$ are expressed with respect to the indicated reference frames. Thus the modular relative Jacobian as derived in [10] can be expressed as

$$
\mathbf{J}_{R}=\left[\begin{array}{ll}
-{ }^{2} \Psi_{3}{ }^{2} \Omega_{1} \mathbf{J}_{A} & { }^{2} \Omega_{4} \mathbf{J}_{B}
\end{array}\right]
$$

where

$$
{ }^{2} \Psi_{3}=\left[\begin{array}{cc}
\mathbf{I} & -S\left({ }^{2} \mathbf{p}_{3}\right) \\
\mathbf{0} & \mathbf{I}
\end{array}\right] \quad \text { and } \quad{ }^{i} \Omega_{j}=\left[\begin{array}{cc}
{ }^{i} \mathbf{R}_{j} & \mathbf{0} \\
\mathbf{0} & { }^{i} \mathbf{R}_{j}
\end{array}\right] .
$$

The matrix ${ }^{2} \Psi_{3}$ is the wrench transformation matrix expressed in the indicated reference frames, while the matrix
${ }^{i} \Omega_{j}$ is a rotation matrix in the corresponding reference frames. The symbol $S(\mathbf{p})$ is a skew-symmetric matrix with input vector $\mathbf{p}$, that is,

$$
S(\mathbf{p})=\left[\begin{array}{ccc}
0 & -p_{z} & p_{y} \\
p_{z} & 0 & -p_{x} \\
-p_{y} & p_{x} & 0
\end{array}\right]
$$

Previous expressions [11]-[13] of the modular relative Jacobian $\mathbf{J}_{R}^{\prime}$ assumes the wrench transformation matrix ${ }^{2} \Psi_{3}$ to be an identity matrix. In this work, we compare the dual-arm force and motion control performance in using $\mathbf{J}_{R}$ and $\mathbf{J}_{R}^{\prime}$.

For mobile manipulators, the relative Jacobian $\mathbf{J}_{R T}$ was derived in [10] as,

$$
\mathbf{J}_{R T}=\left[\begin{array}{llll}
-{ }^{2} \Psi_{3}{ }^{2} \Omega_{0} \mathbf{J}_{b A} & -{ }^{2} \Psi_{3}{ }^{2} \Omega_{1} \mathbf{J}_{A} & { }^{2} \Psi_{3}{ }^{2} \Omega_{0} \mathbf{J}_{b B} & { }^{2} \Omega_{4} \mathbf{J}_{B}
\end{array}\right]
$$

where $\mathbf{J}_{b A}$ is the Jacobian of the mobile base $A$ and $\mathbf{J}_{b B}$ is the Jacobian of the mobile base $B$. The frame assignment is the same as in Fig. 2 but the frames $\mathbf{S}_{1}$ and $\mathbf{S}_{4}$ are now moving.

\section{IMPLEMENTATION}

To implement the proposed relative Jacobian to the KUKA dual-arm, we define the corresponding terms in (1). We denote the position and orientation of the robot $A$ endeffector with respect to its own base as $\mathbf{p}_{A}, \mathbf{R}_{A}$; and the robot $B$ end-effector position and orientation with respect to its own base as $\mathbf{p}_{B}, \mathbf{R}_{B}$. Then the following assignments are true: ${ }^{1} \mathbf{R}_{2}=\mathbf{R}_{A},{ }^{1} \mathbf{p}_{2}=\mathbf{p}_{A},{ }^{4} \mathbf{R}_{3}=\mathbf{R}_{B}$, and ${ }^{4} \mathbf{p}_{3}=\mathbf{p}_{B}$.

From (2), we can get

$$
{ }^{2} \Omega_{3}=\left[\begin{array}{cc}
\mathbf{R}_{A}^{T} & \mathbf{0} \\
\mathbf{0} & \mathbf{R}_{A}^{T}
\end{array}\right] \text { and }{ }^{2} \Omega_{4}=\left[\begin{array}{cc}
\mathbf{R}_{A}^{T}{ }^{1} \mathbf{R}_{4} & \mathbf{0} \\
\mathbf{0} & \mathbf{R}_{A}^{T}{ }^{1} \mathbf{R}_{4}
\end{array}\right]
$$

and from [10], we can express the relative position between the end-effectors ${ }^{2} \mathbf{p}_{3}$ as

$$
{ }^{2} \mathbf{p}_{3}=\mathbf{R}_{A}^{T{ }^{1}} \mathbf{p}_{4}+\mathbf{R}_{A}^{T{ }^{1}} \mathbf{R}_{4} \mathbf{p}_{B}-\mathbf{R}_{A}^{T} \mathbf{p}_{A}
$$

where the reference frames $\mathbf{S}_{1}$ and $\mathbf{S}_{4}$ (function of ${ }^{1} \mathbf{p}_{4},{ }^{1} \mathbf{R}_{4}$ and are normally static) are dependent on how robots $A$ and $B$ are mounted on the shoulders of the torso.

\section{A. Torso Mounting}

The shoulder mounting of our torso has a 60-degree inclination from the horizontal and another 30-degree rotation around the normal to the inclined plane. From this shoulder configuration, the orientation ${ }^{1} \mathbf{R}_{4}$ and position ${ }^{1} \mathbf{p}_{4}$ are defined as

$$
{ }^{1} \mathbf{R}_{4}=\mathbf{R}_{z,-30} \mathbf{R}_{y,-120} \mathbf{R}_{z, 150}
$$

and

$$
{ }^{1} \mathbf{p}_{4}=\mathbf{R}_{z,-30} \mathbf{R}_{y,-150} \mathbf{p}_{\text {bases }}
$$

where $\mathbf{p}_{\text {bases }}=[0,0,0.22]^{T} \mathrm{~m}$. The symbol $\mathbf{R}_{i, a}$ denotes rotation of $a$ degrees around the $i$-th axis. 


\section{B. Gravity Compensation}

Due to the change in the configuration of the base mounting, the KUKA robot need to be corrected in terms of the its gravity compensation vector $\mathbf{g}=[0,0,9.81]^{T}$. The modified gravity compensation for the right arm is

$$
\mathbf{g}_{\text {right }}=\mathbf{R}_{z, 30} \mathbf{R}_{y,-60} \mathbf{g}
$$

and the left arm is

$$
\mathbf{g}_{\text {left }}=\mathbf{R}_{z,-30} \mathbf{R}_{y,-60} \mathbf{g}
$$

This results into $\mathbf{g}_{\text {right }}=[-7.36,-4.25,4.91]^{T}$ and $\mathbf{g}_{\text {left }}=[-7.36,4.25,4.91]^{T} \mathrm{~m} / \mathrm{s}^{2}$.

\section{Torque Controller}

We use the KUKA Controller 30, defined as each-axis controller. To implement a force and motion controller on the KUKA, its impedance controller is given as

$$
\tau_{c m d}=\mathbf{J}^{T}\left(\mathbf{k}_{c}\left(\mathbf{x}_{F R I}-\mathbf{x}_{m s r}\right)+\mathbf{F}_{F R I}\right)+D\left(\mathbf{d}_{c}\right)+f_{\text {dynamics }}(\mathbf{q}, \dot{\mathbf{q}}, \ddot{\mathbf{q}})
$$

where $\tau_{c m d}$ is the commanded torque to the robot joints, $\mathbf{J}^{T}$ is the manipulator Jacobian, $\mathbf{k}_{c}$ is the motion control proportional gain, $\mathbf{x}_{F R I}$ is the desired end-effector position and orientation, $\mathbf{x}_{m s r}$ is the measured end-effector position and orientation, $\mathbf{F}_{F R I}$ is the force/torque input, $\mathbf{d}_{c}$ is the motion damping gain, and $f_{\text {dynamics }}(\mathbf{q}, \dot{\mathbf{q}}, \ddot{\mathbf{q}})$ is the robot dynamics model. From the variables in (11), the fast research interface (FRI) of KUKA (which is its programming interface), only allows modification of $\mathbf{k}_{c}, \mathbf{x}_{F R I}, \mathbf{F}_{F R I}$, and $\mathbf{d}_{c}$.

To overwrite the KUKA controller, we set $\mathbf{x}_{F R I}=\mathbf{x}_{m s r}$ (or $\left.\mathbf{k}_{c}=\mathbf{0}\right)$. The force and motion controller is then assigned to $\mathbf{F}_{F R I}$, and the values of $\mathbf{d}_{c}$ are set at the default values. From (11), the resulting KUKA controller then becomes

$$
\tau=\mathbf{J}^{T} \mathbf{F}_{F R I}
$$

such that (11) becomes

$$
\tau_{c m d}=\tau+D\left(\mathbf{d}_{c}\right)+f_{\text {dynamics }}(\mathbf{q}, \dot{\mathbf{q}}, \ddot{\mathbf{q}}) .
$$

Thus for each robot $A$ and $B$ manipulators, the corresponding KUKA controller is set to $\tau_{A}=\mathbf{J}_{A}^{T} \mathbf{F}_{F R I_{-} A}$ and $\tau_{B}=\mathbf{J}_{B}^{T} \mathbf{F}_{F R I \_B}$. These terms are then added to their corresponding damping and dynamics terms to get the commanded torque for each manipulator. To implement the dual-arm controller, the controller torques for both robots are set at,

$$
\left[\begin{array}{l}
\tau_{A} \\
\tau_{B}
\end{array}\right]=\mathbf{J}_{R}^{T} \mathbf{F}_{R}+\left(\mathbf{I}-\mathbf{J}_{R}^{T} \mathbf{J}_{R}^{T+}\right)\left[\mathbf{J}_{A} \mathbf{0}\right]^{T} \mathbf{F}_{N}
$$

where $\mathbf{F}_{R}$ is the controller for both motion and force in the relative operational space, and $\mathbf{F}_{N}$ is the motion controller in the null space.
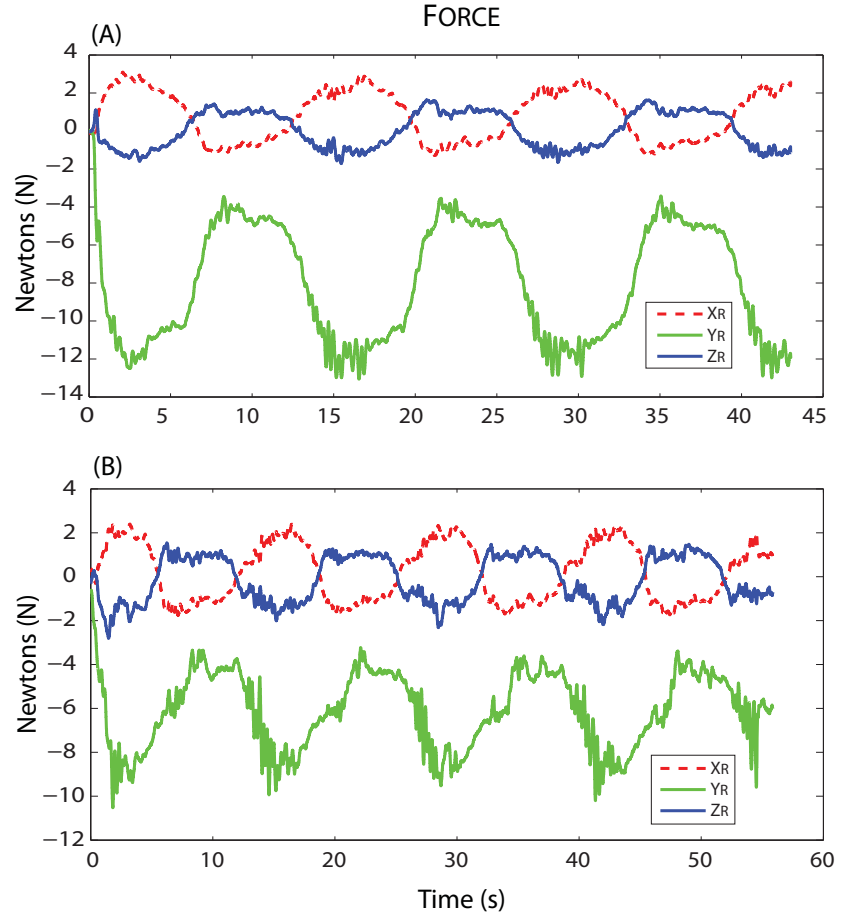

Fig. 3. Force sensor feedback expressed at the reference end-effector frame. The normal force exerted by the tool end-effector is along the $\mathbf{y}_{R^{-}}$ axis, while both end-effectors are moving horizontally along the table in a relative motion with respect to each other. Case $\mathbf{J}_{R}$ is shown in subfigure A, while case $\mathbf{J}_{R}^{\prime}$ is shown in subfigure B.

\section{EXPERIMENT I: Relative END-EFFECTOR Motion}

The KUKA dual-arm platform posed two major challenges to our force and motion control experimental results. First, it does not give access to any low-level real-time controller. And second, to simultaneously control the two robots, we can only achieve a maximum sampling frequency $125 \mathrm{~Hz}$ $(8 \mathrm{~ms})$. Because of these limitations, the force and motion controller gains were not optimally tuned. However given these constraints, the performed experiments still showed comparative results (although non-optimal) between the use of $\mathbf{J}_{R}$ and $\mathbf{J}_{R}^{\prime}$ that substantiate the effect of the wrench transformation matrix in the force control performance. A hybrid force/motion controller is implemented all throughout the experiments.

In the first experiment, we refer to Fig. 1 for the controller setup. In the relative operational space, the two end-effectors will move with respect to each other along the table (along $\mathbf{z}_{R}$-axis), while the tool end-effector will exert a normal force on the table (along $\mathbf{z}_{F}$-axis). To achieve this, force is controlled along the relative $\mathbf{y}_{R}$-axis, with a desired force of $-20 \mathrm{~N}$. A desired sinusoidal motion along the relative $\mathbf{z}_{R}$-axis is specified as

$$
z_{R \_d e s}=A(\cos (\omega t)-\cos (\omega t-\phi))
$$

where $A=0.5 \mathrm{~m}$ is the desired amplitude, $\omega=0.15 \pi \mathrm{rad} / \mathrm{s}$ is the desired angular frequency, $t$ is current time, and $\phi=10$ degrees is the desired phase shift, which determines the magnitude of the incremental step size. The remaining 

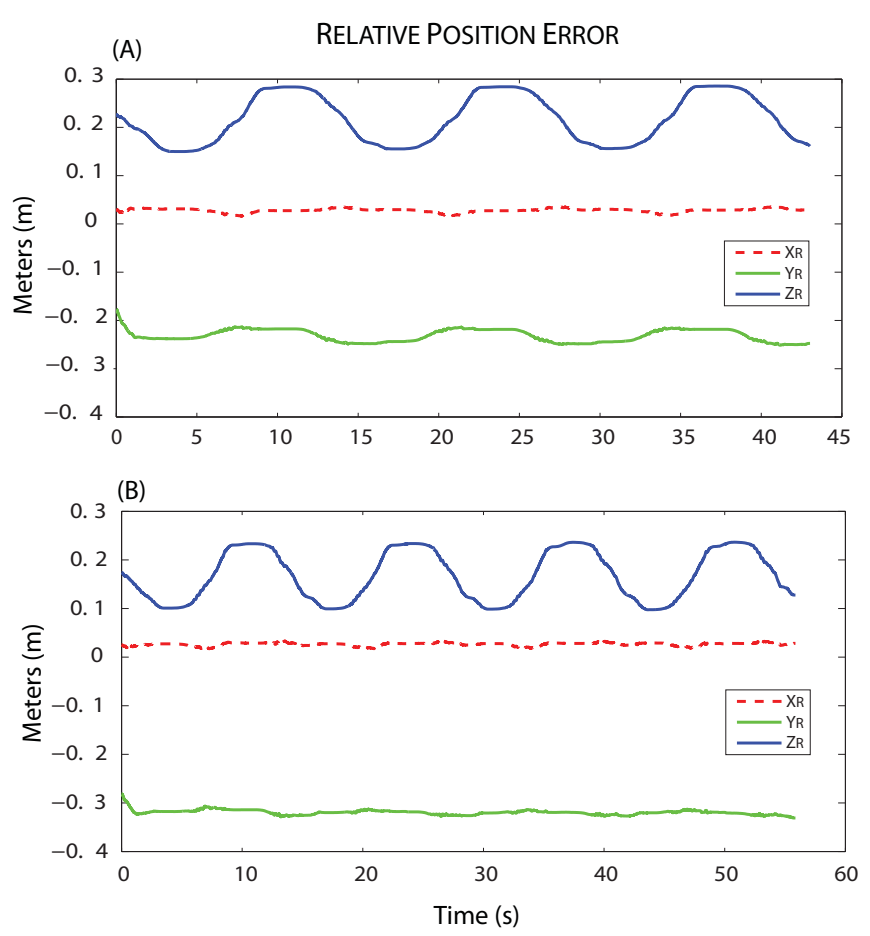

Fig. 4. Relative position error of tool end-effector motion with respect to the reference end-effector: case $\mathbf{J}_{R}$ is shown in subfigure A and case $\mathbf{J}_{R}^{\prime}$ is shown in subfigure B.

relative axes are in motion control, specified to maintain the initial position and orientation. Proportional position gains are set at 200 while proportional orientation gains are at 100. Proportional force gain is set at 0.2 .

Fig. 3 showed the force control performance between $\mathbf{J}_{R}$ (subfigure A) and $\mathbf{J}_{R}^{\prime}$ (subfigure B). The $\mathbf{x}_{R^{-}}$and $\mathbf{y}_{R^{-}}$axes are controlled in motion and are showing force feedback along those directions to be approximately the same. With a desired force along $\mathbf{y}_{R}$ set to $-20 N, \mathbf{J}_{R}$ exerted an average range of around $[-4,-12] N$, while $\mathbf{J}_{R}^{\prime}$ only exerted an average range of around $[-4,-10] N$. A maximum normal force of $-2 N$ difference with $\mathbf{J}_{R}$ case being closer to the desired force. Thus even with a non-optimal controller, the effect of the wrench transformation matrix showed better performance in force control. We believe that at a higher sampling frequency (around $1 \mathrm{kHz}$, that is eight times faster than the current setup), the force control performance comparison can be better.

Relative position error between the tool and reference endeffectors are shown in Fig. 4 for $\mathbf{J}_{R}$ (subfigure A) and $\mathbf{J}_{R}^{\prime}$ (subfigure B). Along the $\mathbf{x}_{R}$-axis, the desired position is the initial position just before the task execution. In both $\mathbf{J}_{R}$ and $\mathbf{J}_{R}^{\prime}$ cases, the error is approximately the same. Along the $\mathbf{y}_{R}$-axis, position is not controlled because this is the force control direction. However, the error value with respect to the initial position can be indicative on how well the force controller is performing, with the $\mathbf{J}_{R}$ case having $0.1 \mathrm{~m}$ less error than $\mathbf{J}_{R}^{\prime}$. Along the $\mathbf{z}_{R}$-axis, the average error of the $\mathbf{J}_{R}$ case is around $0.22 \mathrm{~m}$, while for the $\mathbf{J}_{R}^{\prime}$ case it is around $0.18 \mathrm{~m}$, a difference of around $0.04 \mathrm{~m}$, with $\mathbf{J}_{R}$ case being

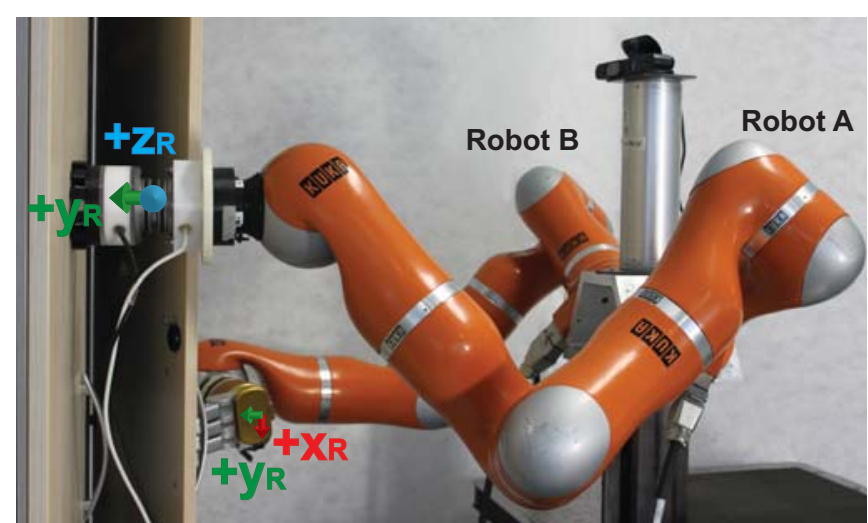

Fig. 5. The KUKA dual-arm manipulator performing a coordinated independent task: robot $B$ opening and closing a cabinet door by moving along the $\mathbf{y}_{R}$, and robot $A$ exerting a normal force (along $\mathbf{y}_{R}$ ) on the other cabinet door while moving along an oscillatory motion along $\mathbf{z}_{R}$.

slight higher. This slightly higher position error in the $\mathbf{J}_{R}$ case can be attributed to the fact that the controller gains were not optimally set and are therefore much more sensitive to noise and disturbances. The higher normal force exertion along the $\mathbf{y}_{R}$-axis in the $\mathbf{J}_{R}$ case created higher friction that resists the motion of the tool end-effector and subsequently, a bigger disturbance to the motion control in the $\mathbf{z}_{R}$ direction, resulting into a slightly higher relative position error along $\mathbf{z}_{R}$.

\section{EXPERIMENT II: COORDINATED INDEPENDENT TASKS}

The second experiment is for robot $B$ to open and close a cabinet door, while robot $A$ exerts a normal force on the other cabinet door and moving in an oscillatory manner. This experiment is designed to show two things: (1) the ease of implementing motion for the reference end-effector, and (2) the capability of the relative Jacobian to show seemingly independent tasks, but were performed through relative motion (or force).

\section{A. Experimental Design}

We note that robot $B$ (reference/gripper) end-effector is in the null-space of the dual-arm manipulator, thus its absolute location can be arbitrary. This makes the opening and closing of the door task much easier to implement. Because the reference end-effector grips the cabinet door handle (and its absolute location is arbitrary), a human can open and close the cabinet door and the reference end-effector will be compliant to this motion, that is, it will keep gripping the cabinet door and the rest of the robot arm will move to accommodate such an end-effector displacement.

The ease of implementation has two aspects. First, we only need to specify the oscillatory displacement along the $\mathbf{y}_{R}$-axis for the gripper end-effector, without specifying any desired displacement along the $\mathbf{z}_{R}$-axis to accommodate the arc movement of the door handle in the operational space. Second, given a specified amplitude of oscillation, the start location of the oscillatory motion can be arbitrary because once the gripper hits the maximum allowable displacement 

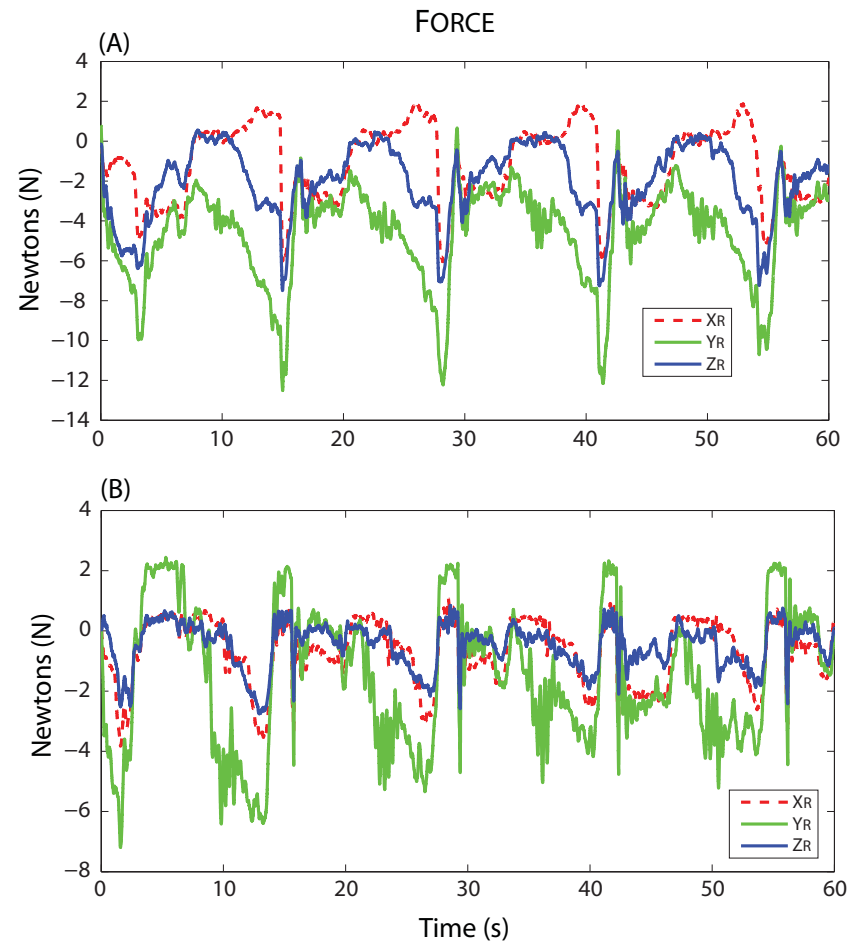

Fig. 6. Force sensor feedback expressed with respect to the reference end-effector frame for the coordinated independent tasks experiment. The normal force exerted on the other cabinet door is along $\mathbf{y}_{R}$. Case $\mathbf{J}_{R}$ is shown in subfigure A and case $\mathbf{J}_{R}^{\prime}$ is shown in subfigure B.

(cannot push further against the cabinet) along the $\mathbf{y}_{R}$-axis, the tool end-effector moves to adjust the relative motion between the two end-effectors. However, the tool end-effector is force controlled (no motion control) along the $\mathbf{y}_{R}$-axis, so it will not move but will keep on exerting a normal force on the other cabinet door.

The second point of the experimental design is the independence of the task execution despite using relative motion between the end-effectors. The tool end-effector is force controlled along the $\mathbf{y}_{R}$-axis, while moving in an oscillatory motion along the $\mathbf{z}_{R}$-axis (compared to the oscillatory movement of the gripper end-effector along $\mathbf{y}_{R}$ ). Thus both tool and gripper end-effector will appear oscillating along two independent axes, while the tool end-effector exerts a normal force on the other cabinet door. Although the tool end-effector's oscillating motion along $\mathbf{z}_{R}$ needs to adjust to the small displacement of the gripper in this direction (due to the arc movement of the door handle), this motion is not critical to the tool end-effector's task execution.

\section{B. Experimental Results}

While the gripper end-effector is opening and closing one cabinet door, the tool end-effector is oscillating on the surface of the other cabinet door and exerting a normal force on the surface. The normal force feedback in shown in Fig. 6. Again, because KUKA does not allow access to a low-level, real-time controller and the sampling frequency is at $125 \mathrm{~Hz}$, the force control is expected to be non-optimal. To keep the tool end-effector to maintain contact during task execution,
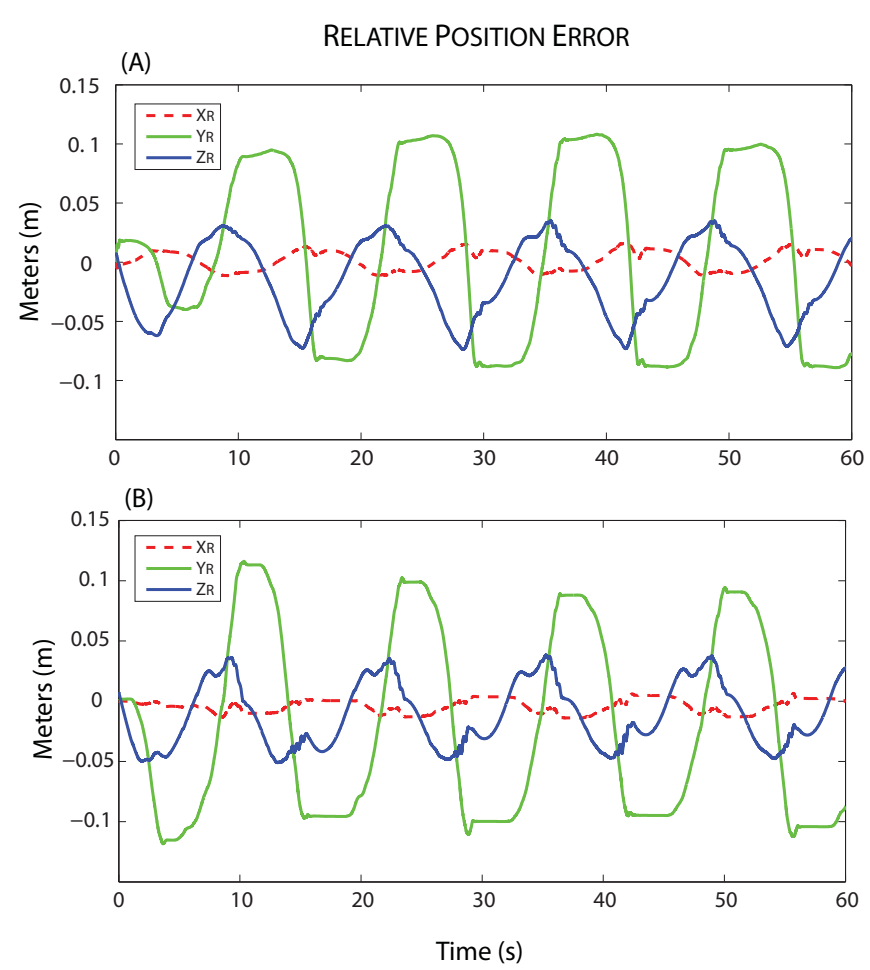

Fig. 7. Relative position errors between tool end-effector and reference end-effector during coordinated independent tasks experiment. The $\mathbf{J}_{R}$ case is shown in subfigure $\mathrm{A}$, while $\mathbf{J}_{R}^{\prime}$ case is shown in subfigure $\mathrm{B}$.

we specify a desired normal force of $-30 \mathrm{~N}$. For case $\mathbf{J}_{R}$, it is able to exert a normal force in the average range of around $[0,-11] N$ (subfigure A), while case $\mathbf{J}_{R}^{\prime}$ is within the average range of around $[2,-5] N$ (subfigure B) and losing surface contact. As seen in these results, with a much more complicated task execution between the end-effectors, a much more superior force control response was shown by $\mathbf{J}_{R}$, exerting around double the maximum force compared to $\mathbf{J}_{R}^{\prime}$. At the same time, the latter case consistently loses contact as indicated by an offset non-contact force of around $2 N$. (This offset force value was set just before task execution, and enhances visualization of the non-contact case. This implies that the actual normal force exerted is slightly higher the force reading. It is noted that the offset is present in both cases, but not necessarily equal in value.) And because $\mathbf{J}_{R}^{\prime}$ case consistently loses contact during the oscillatory motion, it has lesser value of the range of force along the $\mathbf{x}_{R}$ and $\mathbf{z}_{R}$ axes compared to the $\mathbf{J}_{R}$ case.

The relative position errors during the coordinated independent tasks are shown in Fig. 7, with case $\mathbf{J}_{R}$ in subfigure $\mathrm{A}$ and case $\mathbf{J}_{R}^{\prime}$ in subfigure B. The $\mathbf{y}_{R}$-axis is force controlled and is therefore not controlled in position, such that in both cases the position errors along this axis are comparable. The $\mathbf{x}_{R}$ axis is fixed (distance from the floor) during task execution, and so the relative position errors along this axis is comparable for both cases. Lastly, the error range along the $\mathbf{z}_{R}$-axis is slightly higher in $\mathbf{J}_{R}$ than $\mathbf{J}_{R}^{\prime}$. Considering the non-optimality of the controller settings, there is a greater disturbance in $\mathbf{J}_{R}$ because of the greater frictional force 
during oscillatory motion. This is true because of the bigger exertion of the normal force, as well as being consistently in contact with the surface of the door cabinet, as compared to the $\mathbf{J}_{R}^{\prime}$ case that kept on losing contact with the door surface.

\section{CONCLUSION}

This paper has shown the contribution of the wrench transformation matrix to the force control using modular relative Jacobian. It clearly indicated its influence by resulting into a more accurate force control performance even at low endeffector velocities. Two experiments were performed to show the difference in performance with and without the wrench transformation matrix. In both experiments, the tool endeffector was required to exert a normal force on a surface, while both tool and reference/gripper end-effectors moved with respect to each other. Experimental results showed that the case with the wrench transformation matrix included has superior force control performance, with a difference of $2 N$ and $5 N$ in maximum force exerted in each respective experiment. As the task became much more complicated, the superiority of the force control performance with the wrench transformation matrix became much more evident, doubling its maximum exerted force compared to the case without this matrix. Furthermore, this paper also showed the ease of implementing two independent tasks, through the use of relative Jacobian: opening and closing a door on one endeffector, and exerting a normal force with oscillatory motion on the other end-effector. This complicated task execution could have required more accurate task specifications if these tasks were implemented independently with dedicated controllers for each end-effector. Lastly, we believe that the experimental difference will be much more pronounced given better controller setup, that is, with access to low-level, realtime controller and at a much higher sampling frequency.

\section{REFERENCES}

[1] C. Lewis and A. Maciejewski, "Trajectory generation for cooperating robots," in Systems Engineering, 1990., IEEE International Conference on, 1990, pp. 300-303.

[2] C. Lewis, "Trajectory generation for two robots cooperating to perform a task," in Robotics and Automation, 1996. Proceedings., 1996 IEEE International Conference on, vol. 2, Apr 1996, pp. 1626-1631.

[3] C. Smith, Y. Karayiannidis, L. Nalpantidis, X. Gratal, P. Qi, D. V. Dimarogonas, and D. Kragic, "Dual arm manipulation - a survey," Robotics and Autonomous Systems, 2012.

[4] K. Harada, T. Foissotte, T. Tsuji, K. Nagata, N. Yamanobe, A. Nakamura, and Y. Kawai, "Pick and place planning for dual-arm manipulators," in Robotics and Automation (ICRA), 2012 IEEE International Conference on. IEEE, 2012, pp. 2281-2286.

[5] B. Cohen, S. Chitta, and M. Likhachev, "Search-based planning for dual-arm manipulation with upright orientation constraints," in Robotics and Automation (ICRA), 2012 IEEE International Conference on. IEEE, 2012, pp. 3784-3790.

[6] M.-B. Cheng, W.-C. Su, C.-C. Tsai, and T. Nguyen, "Intelligent tracking control of a dual-arm wheeled mobile manipulator with dynamic uncertainties," International Journal of Robust and Nonlinear Control, vol. 23, no. 8, pp. 839-857, 2013.
[7] P. Hebert, N. Hudson, J. Ma, and J. W. Burdick, "Dual arm estimation for coordinated bimanual manipulation," in Robotics and Automation (ICRA), 2013 IEEE International Conference on. IEEE, 2013, pp. $120-125$.

[8] F. Caccavale, V. Lippiello, G. Muscio, F. Pierri, F. Ruggiero, and L. Villani, "Grasp planning and parallel control of a redundant dualarm/hand manipulation system," Robotica, vol. 31, no. 07, pp. 1169 1194, 2013.

[9] C. Rodríguez, A. Montaño, and R. Suárez, "Planning manipulation movements of a dual-arm system considering obstacle removing," Robotics and Autonomous Systems, 2014.

[10] R. S. Jamisola and R. G. Roberts, "A more compact expression of relative jacobian based on individual manipulator jacobians," Robotics and Autonomous Systems, 2014.

[11] P. Chiacchio, S. Chiaverini, and B. Siciliano, "Task-oriented kinematic control of two cooperative 6-dof manipulators," in American Control Conference, 1993, 1993, pp. 336-340.

[12] B. Cao, G. I. Dodds, and G. W. Irwin, "Redundancy resolution and obstacle avoidance for cooperative industrial robots," Journal of Robotic Systems, vol. 16, no. 7, pp. 405-417, 1999.

[13] J. Lee, P. Chang, and R. S. Jamisola, "Relative impedance control for dual-arm robots performing asymmetric bimanual tasks," Industrial Electronics, IEEE Transactions on, vol. 61, no. 7, pp. 3786-3796, 2014.

[14] J.-D. Choi, S. Kang, M. Kim, C. Lee, and J.-B. Song, "Twoarm cooperative assembly using force-guided control with adaptive accommodation,' in Intelligent Robots and Systems, 1999. IROS '99. Proceedings. 1999 IEEE/RSJ International Conference on, vol. 2, 1999, pp. 1253-1258.

[15] L. Ribeiro, R. Guenther, and D. Martins, "Screw-based relative jacobian for manipulators cooperating in a task," in ABCM Symposium Series in Mechatronics. v3, 2008, pp. 276-285.

[16] W. Owen, E. Croft, and B. Benhabib, "A multi-arm robotic system for optimal sculpting," Robotics and Computer-Integrated Manufacturing, vol. 24, no. 1, pp. 92-104, 2008.

[17] R. S. Jamisola, D. N. Oetomo, M. H. Ang, O. Khatib, T. M. Lim, and S. Y. Lim, "Compliant motion using a mobile manipulator: an operational space formulation approach to aircraft canopy polishing," Advanced Robotics, vol. 19, no. 5, pp. 613-634, 2005.

[18] R. S. Jamisola, A. A. Maciejewski, and R. G. Roberts, "Failure-tolerant path planning for kinematically redundant manipulators anticipating locked-joint failures," Robotics, IEEE Transactions on, vol. 22, no. 4, pp. 603-612, 2006.

[19] R. S. Jamisola, P. Kormushev, A. Bicchi, and D. G. Caldwell, "Haptic exploration of unknown surfaces with discontinuities," in Intelligent Robots and Systems (IROS), 2014 International Conference on. IEEE/RSJ, 2014, pp. 1255-1260.

[20] L. A. Weitz, J. Doebbler, K. E. Johnson, and J. E. Hurtado, “Trajectory planning for the cooperative manipulation of a flexible structure by two differentially-driven robots," Journal of Intelligent and Robotic Systems, vol. 58, no. 2, pp. 149-163, 2010.

[21] N. Michael, J. Fink, and V. Kumar, "Cooperative manipulation and transportation with aerial robots," Autonomous Robots, vol. 30, no. 1, pp. 73-86, 2011.

[22] J. Fink, N. Michael, S. Kim, and V. Kumar, "Planning and control for cooperative manipulation and transportation with aerial robots," The International Journal of Robotics Research, vol. 30, no. 3, pp. 324-334, 2011.

[23] W. Li and M. W. Spong, "Decomposition frameworks for cooperative manipulation of a planar rigid body with multiple unilateral thrusters," Nonlinear Dynamics, pp. 1-16, 2014.

[24] S. Erhart, D. Sieber, and S. Hirche, "An impedance-based control architecture for multi-robot cooperative dual-arm mobile manipulation," in Intelligent Robots and Systems (IROS), 2013 IEEE/RSJ International Conference on. IEEE, 2013, pp. 315-322. 